First draft 2001-02-22

Please do not quote

\title{
Incentive and Incarceration Effects in a General Equilibrium Model of Crime
}

\author{
by \\ Mats Persson ${ }^{\mathrm{a}}$ \\ and \\ Claes-Henric Siven ${ }^{\mathrm{b}}$
}

\begin{abstract}
:
An intertemporal general equilibrium model of criminal behavior is used to analyze the effect on crime of changing policy parameters. The policy parameters are the length of the prison term, the severity of punishment, and the amount of police resources. The number of crimes in society can be decomposed into an incentive part, an incarceration part, and a crime competition part. The magnitudes of these three components are studied by means of empirical data from England and the US.

Keywords: Crime, General Equilibrium, Incarceration, Incarceration effect JEL Classification: K42

\footnotetext{
${ }^{a}$ Institute for International Economic Studies, Stockholm University, SE-106 91 Stockholm, Sweden. E-mail: mp@iies.su.se.

${ }^{\mathrm{b}}$ Department of Economics, Stockholm University, SE-106 91 Stockholm, Sweden. E-mail: chs@ne.su.se.
} 


\section{Incentive and Incarceration Effects in a General Equilibrium Model of Crime}

\section{Introduction}

It is common, in the general discussion of crime and punishment as well as in the scholarly literature on the subject, to distinguish between the incentive and the incarceration effect of prison sentence. ${ }^{1}$ This is intuitively natural; a longer prison term is likely both to discourage (the incentive effect) and to disable (the incarceration effect) people from engaging in criminal activities. One reason why these two components of crime are so often discussed is probably that knowledge of their respective magnitudes is important for the evaluation of crime policy. Despite the fact that they are often discussed, however, there is no analytical treatment of them in the literature. There is thus no analytical basis for systematic empirical studies on the subject.

In the present paper, we will provide such an analytical decomposition of crime. Incidentally, we will also derive a third effect, not previously discussed in the literature, which we will call "the crime competition effect". An emerging standard is to treat crime and punishment in a general equilibrium framework ${ }^{2}$ this will be the case in the present paper, too. Also, a meaningful treatment of prison sentences requires an intertemporal model. To the best of our knowledge, there is only one earlier paper in the literature that analyzes crime in an explicitly intertemporal framework. $^{3}$

In virtually all papers on the economics of crime following Becker (1968), people are assumed to differ with respect to their productivity in honest work. Low-productivity individuals will thus choose to become criminals. An interesting feature of the intertemporal approach is that it allows for other assumptions as to what determines people's choice of whether to engage in criminal activities. We have thus assumed that everybody has the same productivity in honest work, but that people differ in

\footnotetext{
${ }^{1}$ Cf. for example Ehrlich (1981), Shavell (1987) and Polinsky and Shavell (2000).

${ }^{2}$ See Fender (1999), Murphy et al. (1993) and Furlong (1987)). In addition, Ehrlich (1973), Carr-Hill and Stern (1973) and Phillips and Votey (1975) use econometric models implying a general equilibrium approach.
} 
terms of time preference. This is technically a minor issue; the model could be solved using the standard assumption (i.e., different productivity and identical rates of time preference) as well. But since the assumption of different time preferences has not been made before, we choose it for the sake of novelty. ${ }^{4}$ Moreover, the two assumptions have slightly different policy implications, and it seems like an interesting avenue for future research to work out the predictions of the two approaches in order to assess their relative degree of realism against empirical data.

The paper is organized as follows. In Section 2, the basic model is presented. Section 3 contains a general characterization of the model by means of numerical simulations. In section 4 we discuss the decomposition of crime into the incentive, incarceration and crime competition effects, and provide some numerical illustrations of their magnitudes. Section 5 concludes the paper.

\section{The Model}

\subsection{Individual Utilities}

In each time period, an honest worker will earn a gross income $w$. Everybody has the same wage rate, and the honest individual's net earnings are thus $w(1-t)$, where $t$ is the tax rate. Working also inflicts some disutility on the individual. With an inelastic labor supply, we could set the monetary equivalent of this disutility equal to a constant $a$, which is the same for everybody. With a linear utility function, ${ }^{5}$ the instantaneous utility from work is thus $w(1-t)-a$.

\footnotetext{
${ }^{3}$ Davis (1988). That model is however not general equilibrium, and the approach is quite different from ours.

${ }^{4}$ Some writers have observed that in reality, criminals seem to have a very strong preference for immediate gains as compared to future costs (see, for instance, Dilulio, 1996). This has sometimes been interpreted as a contradiction to Becker's approach to crime, indicating that criminals are simply irrational. However, it can equally well be interpreted as a high rate of time preference, which is perfectly compatible with rational behavior.

${ }^{5}$ Although risk aversion is a relevant concept in this setup, where workers as well as criminals are subject to risk, we will disregard this complication and assume risk-neutral agents. For discussions of preferences as to risk in connection with crimes, see Becker (1968), Block and Heinecke (1975) and Ehrlich (1996).
} 
With a probability $\lambda$, the worker will meet a criminal and get robbed of his net earnings ${ }^{6}$ and with a probability $(1-\lambda)$ he will avoid such a fate. The expected instantaneous utility of a worker is thus

$$
E u_{W}=(1-\lambda)[w(1-t)-a]+\lambda(-a) \equiv(1-\lambda) w(1-t)-a .
$$

The $\lambda(-a)$ term stems from the fact that if the worker is robbed, only his disutility of work (which is equal to $-a$ ) remains.

Next period, the person will go to his work again, and since there is no accumulation of capital, there are no intertemporal links; each period will look exactly the same to him as the previous one. This means that a person who has initially chosen to be honest will remain so for the rest of his life. Under stationary conditions, the expected lifetime utility of a worker (where we for simplicity have assumed an infinite life) is thus

$$
V_{W}=(1-\lambda) w(1-t)-a+\delta V_{W},
$$

where $\delta$ is the discount factor. We assume that people differ with respect to their rate of time preference, and that the discount factor $\delta$ is uniformly distributed on $[0,1]$.

One should not take the robbery scenario above too literally. It has been used to lend a factual flavor to the exposition of the model, but the reasoning is in fact more general; it could as well be used to analyze burglary, or fraud, or most other types of crime. For the sake of exposition, however, the encounters between workers and criminals will be described in terms of the robbery story throughout this paper.

For a criminal, life is somewhat more complicated. First, he has to encounter a victim. This happens with a probability $\mu$, and we make the simplifying assumption that a

\footnotetext{
${ }^{6}$ We have assumed that the tax is paid first, before leaving the workplace and running the risk of being robbed. The alternative assumption is perhaps equally plausible and would not change anything in principle in the model.
} 
criminal can meet at most one person per time period. ${ }^{7}$ With probability $(1-\mu)$ he does not encounter any victim, and his utility is then zero for that period.

Given that the criminal has encountered a victim, a robbery takes place. The booty is what the worker carries on him, i.e., the period's net income $w(1-t)$. In the next period, the police catches the robber with probability $p$, in which case he will be sentenced to $n$ periods in prison, each period yielding a utility $-f$. With probability $(1-p)$, he is not apprehended, in which case he can start all over again in the next period: with probability $\mu$ he meets a worker, etc.

The above scenario implies that for the criminal, there is a simple intertemporal link in the model. If he robs a worker, and if he then gets caught by the police (this joint event occurring with probability $\mu p$ ), his next period will not look exactly like the previous one. Instead, he will enjoy $n$ periods in prison. What makes the model simple is, however, that this link is not based on any choice variable; there is no accumulation of capital in the model, but the $n$-period prison term is the result of a random draw only.

The expected lifetime utility of a criminal can thus be expressed as

$$
V_{C}=(1-\mu) \delta V_{C}+\mu\left[w(1-t)+(1-p) \delta V_{C}+p\left(\sum_{i=1}^{n} \delta^{i}(-f)+\delta^{n+1} V_{C}\right)\right]
$$

Solving for $V_{W}$ in (1) and for $V_{C}$ in (2), we set $V_{W}=V_{C}$ to obtain the cut-off discount rate $\hat{\delta}$ at which an individual is indifferent between honest work and criminal activity:

$$
(1-\lambda) w(1-t)-a=\frac{\mu\left(w(1-t)(1-\hat{\delta})-f p \hat{\delta}\left(1-\hat{\delta}^{n}\right)\right)}{1-\hat{\delta}+\mu p \hat{\delta}\left(1-\hat{\delta}^{n}\right)}
$$

\footnotetext{
${ }^{7}$ In continuous time, this assumption is equivalent to assuming that encounters are Poisson distributed. Since our model is cast in discrete time, such an assumption is less obvious, but we nevertheless make it, for the sake of simplicity. As for the workers, there is no need to assume that they get robbed at most once per time period; since they lose their entire net wage if an encounter takes place, it does not matter whether they later, after having been robbed, happen to meet one more robber.
} 
This is an $(n+1)$ th order equation in the unknown $\hat{\delta}$. For given probabilities $\lambda, \mu$ and $p$, it gives us the number of $\operatorname{criminals}^{8} \hat{\delta}$ and the number of workers $(1-\hat{\delta})$.

\subsection{The Endogenous Probabilities}

The traditional approach in the literature since Becker (1968) has been to regard crime as a risky activity; with probability $p$ the criminal is punished, and with probability $(1-p)$ he gets away with his deed. Honest work, on the other hand, is usually assumed to be a safe activity. The implication of this, if one assumes risk-averse individuals, is that ceteris paribus, people with a low degree of risk aversion will choose to be criminals. We think this is not an altogether correct view of the world. In reality, being a worker will be risky, too. In fact, many honest people complain of the fear and the risk they perceive from high crime rates in their neighborhood. While we, in our model, will not model risk aversion explicitly, we will model the fact that being a worker is risky, too.

The probability that a worker will be robbed, $\lambda$, depends on the encounter technology. Basically, the probability should be increasing in the number of criminals, and decreasing in the number of workers. There are many ways to model such an encounter technology, the simplest approach being to assume that in each period, a worker meets exactly one person in the population, picked at random. If that person is a criminal, the worker gets robbed.

The number of criminals in the population is $\hat{\delta}$. This group consists of $i$ persons in prison (internees) and $\hat{\delta}-i$ persons outside prison (externees). Only the latter constitute any danger to the workers. The total number of people in the streets is $(1-\hat{\delta})+(\hat{\delta}-i)$, and thus the probability that the person you meet in the street is a criminal is

\footnotetext{
${ }^{8}$ If one thinks the assumption of $\delta$ having a uniform distribution on $[0,1]$ is rather special, it should be noted that the switchpoint condition (3) holds regardless of the distribution. The only difference is that with a uniform distribution, $\hat{\delta}$ could be directly interpreted as the number of criminals in society,
} 


$$
\lambda=\frac{\hat{\delta}-i}{(1-\hat{\delta})+(\hat{\delta}-i)}=\frac{\hat{\delta}-i}{1-i}
$$

The number of internees is, in stationary state, equal to the number of criminals caught during a period, which is equal to $p \mu(\hat{\delta}-i)$, times the average term in prison: $i=p \mu(\hat{\delta}-i) n$. This gives us the number of internees as

$$
i=\hat{\delta} \frac{p \mu n}{1+p \mu n}
$$

Substituting this into the expression for $\lambda$, we obtain

$$
\lambda=\frac{\hat{\delta}}{1+(1-\hat{\delta}) p \mu n} .
$$

Let us now turn to the criminal, i.e., the externee. Like in the case of the worker, we assume that the criminal meets only one person per period of time. If he meets another externee, nothing happens. If he meets a worker, a robbery takes place. The number of potential victims is thus $(1-\hat{\delta})$ out of a total number of persons in the streets being equal to $(1-\hat{\delta})+(\hat{\delta}-i)=1-i$. Thus the probability that the person he meets is a worker is

$$
\mu=1-\lambda
$$

As for $p$, the probability of apprehension, we have to assume an apprehension technology. There are several alternatives, none of which is more realistic on a priori grounds than the others.

One possible apprehension technology is based on random encounters between policemen and (guilty) criminals, just like the probability of being robbed depended

whereas for an arbitrary distribution, there exists a one-to-one relation between $\hat{\delta}$ and the number of criminals in society. Note that the population has been normalised to 1 . 
on random encounters between workers and (not yet guilty) criminals. Thus the probability $p$ could be equal to the ratio of the number of policemen to the total population in the streets, consisting of (robbed and non-robbed) workers, (successful and unsuccessful) criminals, and policemen. This apprehension technology is quite possible to model within our framework.

An alternative is to assume that the probability of apprehension depends on the number of crimes per policeman. While the previous technology was based on the image of policemen randomly strolling in the streets, this technology instead is based on the image of a limited number of police investigators being burdened with a large amount of paperwork. Each paper corresponds to one reported crime, and the larger the number of papers, the smaller the probability that a particular case will be satisfactorily solved. For the time being, we have chosen this technology. Letting $\chi$ denote the number of crimes per policeman, we can thus express the probability of apprehension as

$$
p=\varphi(\chi) \equiv \varphi\left(\frac{\text { number of crimes committed }}{\text { number of policemen }}\right)
$$

For the function $\varphi(\chi)$, any function taking values between zero and unity is possible; in our applications, we have chosen the exponential function. It turns out that the number of crimes per policeman can be expressed on a very simple form in our model.

Policemen are paid for by taxes. Total tax revenue is

$$
t \int_{\hat{\delta}}^{1} w d F(\delta) \equiv t \cdot w \cdot(1-\hat{\delta})
$$

Assuming that a policeman has the same wage rate as a worker, tax revenue is sufficient to hire $t \cdot(1-\hat{\delta})$ policemen. $^{9}$

\footnotetext{
${ }^{9}$ For simplicity, we assume that there is no cost associated with keeping internees in prison. In order to avoid too many choices of occupation, we have also assumed that the policemen are hired from
} 
The number of crimes per period, $c$, is equal to the number of externees, times their probability of encounter:

$$
c=(\hat{\delta}-i) \mu=\frac{\hat{\delta} \mu}{1+p \mu n} .
$$

If we divide the number of crimes, given by (8), by the number of policemen, given by (7), we can thus write the number of crimes per policeman as

$$
\chi=\frac{\hat{\delta} \mu}{t \cdot(1-\hat{\delta})(1+p \mu n)}
$$

Now, if we substitute $\lambda$, given by (5), into (4), we can solve the resulting expression for $\hat{\delta}$ and substitute into (9). This gives a surprisingly simple expression for the number of crimes per policeman:

$$
\chi=(1-\mu) / t
$$

Thus the probability of apprehension is $p=\varphi((1-\mu) / \alpha t)$, where $\alpha$ is a productivity parameter. If we use the exponential function, this becomes

$$
p=\exp \left(-\frac{1-\mu}{\alpha t}\right)
$$

The importance of the endogenous probabilities $\lambda, \mu$ and $p$ should be emphasized. A few authors, like Sah (1991) and Fender (1999), have recognized that an increase in the total number of criminals in society will reduce, cetiris paribus, the probability that an individual criminal be arrested. Thus a larger number of criminals can make

somewhere outside the model economy. An alternative interpretation is that every honest person works a fraction $(1-t)$ of his time at his ordinary job, and a fraction $t$ at the police station. In principle, it would not be a problem to let the population consist of three groups: policemen, workers, and criminals. This would however only complicate the model without yielding any additional insights. As 
crime a more profitable activity. The other side of the coin, which we want to stress, is that a larger number of criminals means more competition for the individual crook; thus the probability $\mu$ will fall. The net effect of the fall in $p$ (which makes crime more profitable) and the fall in $\mu$ (which makes crime less profitable) has to be determined in a general equilibrium model.

Equations (3), (4), (5) and (11) constitute the general equilibrium model ${ }^{10}$ in the four endogenous variables $\hat{\delta}, \lambda, \mu$, and $p$. By successive substitutions the whole system can be ultimately reduced to one equation in $\mu$. The exogenous variables are the technological parameters $w, a$, and $\alpha$ together with the policy variables. We have three policy variables: the tax rate $t$, the punishment $f$, and the length of the prison term, $n$. Since the model is highly non-linear we must rely on numerical simulations in order to derive its properties.

\section{Some Numerical Simulations}

When solving this model numerically, we do not mainly aim at realism, but rather at providing illustrations of some basic mechanisms in the model. Still, we do not want to use utterly unrealistic parameter values. Our benchmark parameter values are the following: $w=5, a=1, \alpha=5, f=0, t=0.05$ and $n=10$. This leads to a (stable) solution where $\hat{\delta}=0.2481$, that is, 25 percent of the population choose to be criminals $^{11}$.

We can now analyze how the model's solution varies with the policy parameters at the government's disposal, i. e. with $n, f$ and $t$. In Figure $l$ we show $\hat{\delta}$ for different values of the prison term, holding $f$ and $t$ constant at 0 and 0.05 , respectively. In general, the model has three roots, two of which are stable and one, which is unstable. ${ }^{12}$ The stable roots are depicted by the solid curves in the figure, while the

a consequence of hiring policemen from outside the model, the government's wage cost for hiring them is $w$ rather than $w(1-t)$.

${ }^{10}$ Evidently we assume that agents have perfect information about the endogenous variables. For a discussion of the learning process in crime models, see Sah (1991). Compare also the stability analysis in the Appendix.

${ }^{11}$ The (stable) benchmark solution for the other endogenous variables are $\mu=0.97$ and $p=0.87$.

12 The notion of stability in a model like this is not straightforward. It depends on the assumptions one make regarding the behavior of the agents outside equilibrium. For a discussion of stability, see the Appendix. 
unstable roots are depicted by a dashed curve. The vertical arrows, pointing towards the solid curves and away from the dashed curve, illustrate the out-of-equilibrium dynamics of the model.

There can thus be three equilibria in society. First, there is a high-crime equilibrium with $\hat{\delta}=1$, i.e., everybody is a criminal. That this constitutes an equilibrium can be intuitively understood: if everybody is a criminal, the probability that one will be robbed, given that one is a worker, is unity. Thus $\lambda=1$ and $\mu=0$. Since everybody is a criminal, there is no tax base to pay for the police, and thus $p=0$. The utility from being a worker, who will be robbed with certainty, is zero. But since there are no victims around, the utility of a criminal is also zero. Thus $V_{C}=V_{W}$ and we have an equilibrium. It can also be shown that this equilibrium must be stable. ${ }^{13}$

We then have two interior equilibria: an unstable high-crime one where 40-60 percent of the population are criminals, and a stable low-crime one where 10-30 percent are criminals. It is interesting to note that these two equilibria only occur for the term in prison being above a given minimum value. In this case the critical value is $n_{\min }=5.9$. Thus, as long as the prison term is longer than 5.9 time periods, society can be at a stable low-crime equilibrium with $\hat{\delta}$ at around 10-30 percent (at the borderline case of $n=5.9, \hat{\delta}=0.34$ ). If however the prison term is further reduced, the crime rate will make a discrete jump to the other stable equilibrium of $\hat{\delta}=1$. Without claiming that the numbers are realistic, this feature of the model nevertheless sheds some light on the mechanics behind an observation sometimes made in the real world: small changes in some parameter can cause a dramatic change in society.

Note that the value of $n_{\min }$ depends on the parameter values chosen, i. e., in this case, $t=0.05$ and $f=0$. For other values of these parameters, $n_{\min }$ changes accordingly. One could easily trace $n_{\min }$ as a function of $t$ and $f$, thereby illustrating the policy

\footnotetext{
${ }^{13}$ In the Fender (1999) model, there are also three solutions: one interior, stable, high-crime equilibrium; one interior, unstable, low-crime equilibrium; and one stable equilibrium where the number of criminals equals zero. Inspection of equations (3)-(5) and (11) shows that our model also can produce an equilibrium with $\hat{\delta}=0$. However, this equilibrium exists only if $a=0$.
} 
options open to society. ${ }^{14}$ This would result in a surface in three-dimensional space ( $n$, $f, t$ ) such that all points above the surface would yield interior equilibria $\hat{\delta}<1$, while all points below the surface would yield only a high-crime equilibrium $\hat{\delta}=1$.

The shape of the solution set in Figure 1 of course depends on the parameter values chosen. To check the general characteristics of the model, we have tried many other combinations of $w, a, \alpha, f$ and $t$, and we have obtained the same basic shape of the solution set as the one depicted in Figure 1. Further, in that figure we have solved $\hat{\delta}$ for different values of $n$; it is of course possible to keep $n$ constant (for example at $n=$ 10) and see how $\hat{\delta}$ varies for different values of $f$, or different values of $t$.

So far, we have used the term "crime rate" as synonymous to $\hat{\delta}$. Our model does however allow for a richer interpretation of what we actually mean by crime. We have the following four definitions, all of which are relevant for policy:

(i) The number of criminals in society, $\hat{\delta}$

(ii) The probability that a worker will be robbed, $\lambda$

(iii) The number of criminals in the streets, $\hat{\delta}-i$

(iv) The number of crimes committed, $c$.

An isoquant diagram is a suitable tool for illuminating the importance of keeping track of the different definitions of crime. In Figure 2 we have drawn the isoquants, i. e., the combinations of $n$ and $f$ for which crime is constant. The solid curves correspond to a constant number of criminals ( $\hat{\delta}=0.20$ and 0.25 , respectively), while the dashed curves correspond to a constant number of crimes $(c=0.02$ and 0.03 , respectively). A higher isoquant means a lower level of crime. Note that the $c$ isoquants are steeper than the $\hat{\delta}$ isoquants. This means that if we move to the left along the $\hat{\delta}$ isoquant (that is, we reduce the prison term but increase the punishment $f$ so as to keep $\hat{\delta}$ constant) this policy will actually imply an increasing number of crimes committed. Similarly, if we move to the left along the $c$ isoquant, the number of crimes will remain constant but the number of criminals will fall. Needless to say,

\footnotetext{
${ }^{14}$ These calculations are straightforward and therefore not presented in the paper.
} 
it is also easy to obtain policy changes in $(n, f)$ space for which crime increases according to one definition, while crime decreases according to the other definition.

\section{The Decomposition}

\subsection{Basic Principles}

Several authors have emphasized the need for distinguishing between the incentive and the incarceration effect of extending the duration of terms in prison. However, the literature on these two effects is mainly verbal, and there is no analytical treatment of them. We will now provide an analytical derivation of those effects within the context of our model. We also show that there is a third effect (the "crime competition effect") which so far has been disregared in the literature.

Since there are several definitions of crime we have to decide which one we want to start from. Probably most authors have had the number of crimes per period in mind, so this will be our starting point. In equation (8) we saw that the number of crimes per

period can be written as $c=\hat{\delta} \cdot \frac{1}{1+p \mu n} \cdot \mu$. This is the decomposition we want to use; the incentive effect is represented by the term $\hat{\delta}$, i.e., the number of persons who prefer criminal activity to honest work. The incarceration effect is represented by the middle term, which is the fraction of externees among the criminals. Finally, the term $\mu$ is the probability that a given criminal will find a suitable victim; with more externees in the streets, the competition among them for suitable victims will be harder, thus the term "the crime competition effect". The number of crimes per period can be decomposed into these three terms, which means that a change of the length of imprisonment (or any other parameter change for that matter) affects the number of crimes via three channels: the incentive effect, the incarceration effect, and the crime competition effect. The three effects are not independent, since their magnitudes are simultaneously determined within the general equilibrium model. Still, it can be conceptually interesting to discriminate among them.

To get a starting point for the following discussion we now take the logarithms of both sides of (8) and then differentiate w. r. t. the logarithm of $n$. We consequently 
calculate the elasticity of the number of crimes w. r. t. the prison term. We then divide this elasticity into three components, the incentive, incarceration and crime competition effects. This yields the following expression for the elasticity of the number of crimes per period w. r. t. the prison term:

$$
\frac{\partial c}{\partial n} \frac{n}{c}=\frac{\partial \hat{\delta}}{\partial n} \frac{n}{\hat{\delta}}+\frac{\partial\left(\frac{1}{1+p \mu n}\right)}{\partial n} \frac{n}{\left(\frac{1}{1+p \mu n}\right)}+\frac{\partial \mu}{\partial n} \frac{n}{\mu}
$$

It should be stressed that the various derivatives are general equilibrium derivatives. The same holds for the corresponding elasticities. We consequently compare the values of the endogenous variables in the new equilibrium to the values in the old general equilibrium solution when $n$ increases by an infinitesimal amount. In the next step we illustrate the numerical order of the various elasticities by letting $n$ vary from 6 to 100. The exogenous variables have the same bench-mark values as before: $w=5$, $a=1, \alpha=5, f=0$ and $t=0.05$. We only compare stable low-crime equilibria.

Table 1. Incentive, incarceration and crime competition effects of longer sentences.

\begin{tabular}{|r|r|r|r|r|l|}
\hline$n$ & & $\begin{array}{l}\text { Elasticity of } \\
c \text { w. r. t. } n\end{array}$ & $\begin{array}{l}\text { Incentive effect: } \\
\text { elasticity of } c \\
\text { w. r. t. } n\end{array}$ & $\begin{array}{l}\text { Incarceration } \\
\text { effect: } \\
\text { elasticity of } c \\
\text { w. r. t. } n\end{array}$ & $\begin{array}{l}\text { Crime competi- } \\
\text { tion effect: } \\
\text { elasticity of } c \\
\text { w. r. t. } n\end{array}$ \\
\hline 6 & 0.060313 & -4.133673 & -2.013091 & -2.615069 & 0.494487 \\
\hline 10 & 0.025395 & -1.266534 & -0.215892 & -1.097367 & 0.046725 \\
\hline 15 & 0.015676 & -1.134212 & -0.115762 & -1.042769 & 0.024319 \\
\hline 20 & 0.011389 & -1.089947 & -0.079765 & -1.026724 & 0.016542 \\
\hline 100 & 0.002135 & -1.013242 & -0.012748 & -1.002720 & 0.002226 \\
\hline
\end{tabular}

Table 1 starts at $n=6$ (note that there is no interior equilibrium for $n=5$ at the given parameter values). We first notice that the number of crimes will go down as $n$ increases. This is rather self-evident. We further note that the elasticity of the number 
of crimes per period w. r. t. the prison term decreases in absolute value as $n$ increases and tends to -1 in the limit.

The elasticity of the number of crimes per period w. r. t. the prison term can be decomposed into three parts representing incentive, incarceration and crime competition effects. We see that the absolute value of the incentive effect elasticity is large at $n=6$, but falls rather fast with higher $n$. As mentioned above, this is due to the fact that fewer criminals means that the marginal criminal has a lower discount rate $\delta$, i.e., he is more impatient. Thus he would be less and less discouraged by a longer prison term. This would not be the case if we had used the standard set-up, where all individuals have the same rate of time preference but differ with respect to productivity in honest work. In fact, this is a feature that would make it possible to empirically discriminate between our setup and the standard one.

The numerical value of the incarceration effect elasticity also falls, but not as fast. The reason why it falls is that at large values of $n$, most criminals will already be in jail. When they come out, they will almost immediately be imprisoned again since the number of externees in comparison to the number of policemen is so small. Longer sentences will therefore have smaller and smaller effects on the number of crimes. Finally, an increase in $n$ will have a positive effect on the probability that an externee will commit a crime during the period, but at a decreasing rate. When sentences are prolonged, the number of externees in comparison to workers will fall and this means that the chance for an externee to find a victim will increase. However, when the number of externees is already low, further reductions will have lower effects on crime probabilities.

We finally note that when $n$ gets very large, the number of crimes as well as the incentive and crime probability effect elasticities tend towards zero while the incarceration effect elasticity tends towards -1 . However, it should be remembered that we assume that prisons are self-financing so that prison costs per internee are zero. $^{15}$

\footnotetext{
${ }^{15}$ Throughout this paper, we have assumed that the costs of keeping people in prison are zero. If prison costs are high, we cannot be sure that there exists any interior equilibrium.
} 


\subsection{Empirical Evidence}

Table 1 above relied on numerical simulations of the model. For the three effects to have any relevance for actual policy, there must also be a possibility to empirically assess the three components of $c$. We note immediately that of the variables in (8), $c$ and $n$ are directly observable (we assume that all crimes are reported). Also, $p$ is the percentage of all crimes that are solved; thus $p$ is observable, too. ${ }^{16}$ To be able to empirically decompose $c$ into its three elements, we need to obtain values of the unobservables $\hat{\delta}$ and $\mu$.

This is easily achieved. We solve substitute (5) into (4) and solve for $\hat{\delta}$ :

$$
\hat{\delta}=\frac{(1-\mu)(1+\mu p n)}{1+\mu p n-\mu^{2} p n}
$$

Substituting this into (8) we obtain

$$
c=\left(\frac{(1-\mu)(1+\mu p n)}{1+\mu p n-\mu^{2} p n}\right) \cdot \frac{1}{(1+\mu p n)} \cdot \mu .
$$

Since we know $c$, equation (8') gives us the value of the unobservable $\mu$. We can therefore make the desired decomposition; ${ }^{17}$ equation ( $\left.8^{\prime}\right)$ is the empirically operational form of (8).

It is tempting to insert actual numbers from the real world, in order to asses the three components of crime for different countries, or for different time periods. The purpose of our model is however mainly to provide a consistent framework for analyzing these issues. Many of our assumptions are not sufficiently realistic to lend themselves to immediate empirical work (for example, the assumption of a stationary state; or the assumption of linear utility; or the assumption that a robber can commit at most one

\footnotetext{
${ }^{16}$ We assume that all crimes are reported to the police.

${ }^{17}$ Note that the tax rate $t$ is not necessary for the decomposition. This is a desirable property of the model. In fact, (10) and ( $\left.8^{\prime}\right)$ can be regarded as an equation system in two unknowns, $\mu$ and $t$.
} 
robbery, and that a worker can only be robbed once, per period). ${ }^{18}$ Still, these are mere technical assumptions, which can in principle be dispensed with. Using actual numbers can therefore be illustrative of what our model could in principle be used for, after having been developed to encompass more complexities of the actual world.

With all these caveats expressed, we have inserted into (8') some actual numbers from English and American crime statistics. ${ }^{19}$ The figures refer to robbery. The definition of robbery could of course differ between the two legal systems, but the data are nevertheless intended to be comparable. Since the population in our model is normalized at unity, we have expressed all numbers in per capita terms. The following numbers have been used.

Table 2. Normalized number of robberies per quarter, normalized number of robbers sentenced to prison per quarter, and number of quarters before being released. England and Wales 1981 and 1995, and USA 1981 and 1994. Source: Langan and Farrington (1998, pp. 86, 87, 98 and 99)

\begin{tabular}{|l|l|l|l|l|}
\hline \multirow{2}{*}{} & \multicolumn{2}{l|}{ England and Wales } & \multicolumn{2}{l|}{ USA } \\
\cline { 2 - 5 } & 1981 & 1995 & 1981 & 1994 \\
\hline $\begin{array}{l}\text { Robberies per capita, } \\
c\end{array}$ & $\frac{162,641}{38,724,000 \cdot 4}$ & $\frac{312,871}{41,167,000 \cdot 4}$ & $\frac{1,380,800}{186,336,000 \cdot 4}$ & $\frac{1,298,750}{213,747,000 \cdot 4}$ \\
\hline $\begin{array}{l}\text { Probability of being } \\
\text { incarcerated, } p\end{array}$ & $\frac{3,039}{162,641}$ & $\frac{3,452}{312,871}$ & $\frac{43,471}{1,380,000}$ & $\frac{51,734}{1,298,750}$ \\
\hline Quarters in prison, $n$ & 4.37 & 6.83 & 13.67 & 14.27 \\
\hline
\end{tabular}

\footnotetext{
${ }^{18}$ The correspondence between theoretical concepts and statistical definitions must also be taken into consideration. For example, should $p$ be defined as the number of persons sentenced to prison divided by the number of crimes committed? Or are criminals punished in some other way without being physically imprisoned? Moreover, should the number of crimes be taken from surveys or from police records?

${ }^{19}$ Source: Langan and Farrington (1998, pp 87 and 99). The number of crimes is obtained from victim surveys and defined as the number of offences as a fraction of the population ages 16 or older (for the US 12 or older). According to Languan and Farrington (1998, p. 20) the typical robber in the US commits 4 robberies per year. To make the data compatible to our model, we have therefore set the time period to equal one quarter; that is, the yearly numbers in the data have been divided by 4 . We abstract from the fact that the average number of offenders per crime is somewhat above 1 and that robbers typically commit other crimes for which they may be incarcerated. As time spent in prison we use number of quarters before being released.
} 
The figures on the length of the prison term, $n$, refer to the number of quarters actually served in prison. We thus do not use data on formal sentences; in particular in England, convicts tend to be released long before the formal term has been served.

Inserting these numbers into (8') and solving for $\mu$ yields two roots. One of these is typically close to 1 and the other typically close to 0 . Substituting into (13), the larger root gives a $\hat{\delta}$ close to 0 while the smaller root gives a $\hat{\delta}$ a little below 1 . We choose the former root and get the following decomposition of the total number of crimes into incentive, incarceration and crime competition parts: ${ }^{20}$

Table 3. Empirical decomposition of the normalized number of robberies into incentive, incarceration and crime competition parts. England and Wales 1981 and 1995, and USA 1981 and 1994.

\begin{tabular}{|l|l|l|l|l|}
\hline \multirow{2}{*}{} & \multicolumn{2}{|l|}{ England and Wales } & USA \\
\cline { 2 - 5 } & 1981 & 1995 & 1981 & 1994 \\
\hline $\begin{array}{l}\text { Number of } \\
\text { robberies per capita }\end{array}$ & 0.00105 & 0.00190 & 0.00185 & 0.00152 \\
\hline Incentive part & 0.00123 & 0.00205 & 0.00265 & 0.00238 \\
\hline Incarceration part & 0.85754 & 0.93002 & 0.69956 & 0.63799 \\
\hline $\begin{array}{l}\text { Crime competition } \\
\text { part }\end{array}$ & 0.99895 & 0.99810 & 0.99814 & 0.99848 \\
\hline
\end{tabular}

If we multiply the figures of the three last rows we obtain the (normalized) numbers of robberies. From the incarceration part we note that a larger fraction of the robbers are in circulation in England than in USA. For example, according to Table 3, 85.8 percent of the robbers in England were in circulation in 1981, while only 70 percent of the American robbers were out. This was counteracted by the lower total number of

\footnotetext{
${ }^{20}$ In this section, we refer to the decomposition into the three different parts, not to the incentive, incarceration and crime competition effects. We want to reserve this latter terminology for analyzing the effects of e. g. a change of $n, t$ or $f$ on the total number of crimes, cf. equation (12). To empirically assess these effects would require an econometric analysis.
} 
robbers in England; the English incentive part in England was only half of that in the US. In the mid-1990s, things had changed; now the number of robberies was higher in England than in the US. This was partly due to an increase in the English incentive part, from 0.12 to 0.21 percent. This effect was strengthened by the development of the incarceration part. In the mid-1990s, 93 percent of the English robbers were out, while the corresponding figure was only 63.8 percent for the US.

Note that the crime competition part is close to unity in both countries. This means that for all practical purposes there are no problems for robbers to find a victim within the specified time period. This is what should be expected in a society with a low crime rate. However, if the analysis were geographically disaggregated, we would expect the crime competition part to vary highly between different neighborhoods.

The results in Table 3 referred to levels of the three components. Since the number of crimes has changed over time, it would be interesting to decompose these changes into the three parts. To be sure, the changes may depend on many exogenous factors. Apart from the quite substantial increases in the length of the prison term reported in Table 2, the severity of punishment $(f)$ might have changed, as might the tax rate $(t)$ or the productivity in police work. But regardless of the cause, the change in $c$ can be decomposed into the three effects. Differentiating (8') logarithmically, we obtain

$$
\frac{\Delta c}{c} \approx \frac{\Delta \hat{\delta}}{\hat{\delta}}+\frac{\Delta k}{k}+\frac{\Delta \mu}{\mu},
$$

where $k$ is short for the incarceration component: $k \equiv 1 /(1+p \mu n)$. Using the numbers from Table 3, we obtain the decomposition reported in Table 4 (since the actual numbers have been subject to finite changes, while the decomposition in (14) holds exactly for infinitesimal changes only, there will be small discrepancies in Table 4). 
Table 4: Decomposition of the relative change in the number of crimes between 1981 and 1995 (for the US, 1994).

\begin{tabular}{|l|l|l|}
\hline & $\begin{array}{l}\text { England and Wales: 1981 - } \\
1995\end{array}$ & USA: 1981 - 1994 \\
\hline $\begin{array}{l}\text { Number of robberies } \\
\text { per capita }\end{array}$ & 0.80953 & -0.17838 \\
\hline Incentive part & 0.66666 & -0.10189 \\
\hline Incarceration part & 0.084521 & -0.08801 \\
\hline Crime competition part & -0.00085 & 0.00034 \\
\hline
\end{tabular}

Robberies per capita rose by 80.9 percent in England between 1981 and 1995. This can be decomposed into a 66.7 percent increase in the incentive part and an 8.5 percent increase in the incarceration part. For the US, robberies per capita instead fell by 17.8 percent between 1981 and 1994. Most of this was due to a 10.2 percent fall in the number of criminals, but a fall in the proportion of externees (by 8.8 percent) worked in the same direction.

The decomposition (14) can be done in space as well as in time. We therefore compare the relative difference between England and USA in 1981, and between England and USA in the mid-nineties, respectively: 
Table 5: Decomposition of the relative change in the number of crimes between England (including Wales) and USA in 1981, and England (including Wales) and USA in the mid-nineties, respectively.

\begin{tabular}{|l|l|l|}
\hline & $1981:$ England - USA & 1995 (94): England - USA \\
\hline $\begin{array}{l}\text { Number of robberies per } \\
\text { capita }\end{array}$ & 0.76190 & -0.17838 \\
\hline Incentive part & 1.15447 & 0.16098 \\
\hline Incarceration part & -0.08452 & -0.31400 \\
\hline Crime competition part & -0.00081 & 0.00038 \\
\hline
\end{tabular}

We note that number of robberies per capita was 76.2 percent higher in USA than in England 1981. This is due to an incentive part that is 115 percent higher in the US than in England. The incentive part is partly counteracted by an incarceration part of 8.5 percent; a larger proportion of the robbers are kept in prison in the US than in England. In the middle of the 1990s, things had changed; now England had 17.8 percent more robberies per capita than the US. The difference was accounted for by a drastic fall in the incentive part as compared to the case fifteen years earlier; in the mid-nineties, the incentive part of the US was only 16.1 percent higher than that of England. Instead, the difference between the two countries was mainly accounted for by the fact that the American incarceration part was 31.4 percent lower than the English one.

\section{Conclusions}

Empirical work has to be based on theory. In the case of crime and punishment, theory is right now heading in the direction of general equilibrium models. There is a number of aspects of crime and punishment where time is involved in an essential way. One obvious example is imprisonment. This calls for an intertemporal general equilibrium model of crime. Having done that, concepts like the incentive effect, and the incarceration effect, that have earlier been discussed in verbal terms only, can be given an empirically tractable interpretation. 
There are many other examples where the time element is important for the analysis of crime. Most of these seem to involve the development of human capital, for those outside as well as those inside prison. Also, productivity changes could refer to productivity in ordinary work as well as in criminal activities. A further development of general equilibrium models along these lines seem to be high on the research agenda today. 


\section{Appendix: Stability of Equilibrium}

The solution of the model does not yield a unique equilibrium. Typically there are instead three distinct equilibrium solutions to each configuration of values of the exogenous variables. One of these equilibria implies 100 percent crime; another is characterized by a low level of crime. In addition there is an intermediate-level equilibrium. It turns out that the comparative statics of moving from one low-level equilibrium to another (for example due to a change of $n, f$, or $t$ ) yields the expected comparative-static results; a longer prison term, or a more severe punishment, or more resources to the police, leads to less crime. For medium-level equilibria, however, the endogenous variables generally change in the opposite direction from what one would expect.

To discriminate between the three equilibria we investigate the stability properties. However, it is not self-evident which corresponding dynamic model (in the stable case converging to the particular equilibrium solution) to start from. There are many possibilities and no alternative is a self-evident candidate.

\section{Disequilibrium dynamics}

We have chosen to base the stability analysis on two particular dynamic models. The first one implies that $\hat{\delta}$ is increased or decreased somewhat in comparison to the equilibrium solution. The probabilities $\mu$ and $p$ are correspondingly adjusted so that equations (4), (5) and (11) are still satisfied ${ }^{21}$. The dynamic model then comprises these three equations, and in addition a dynamic version of equation (3): ${ }^{22}$

$$
\dot{\hat{\delta}}=\beta\left[(1-\lambda) w(1-t)-a-\frac{\mu\left(w(1-t)(1-\hat{\delta})-f p \hat{\delta}\left(1-\hat{\delta}^{n}\right)\right)}{1-\hat{\delta}+\mu p \hat{\delta}\left(1-\hat{\delta}^{n}\right)}\right]
$$

\footnotetext{
${ }^{21}$ In his stability analysis Fender (1999) motivates a non-equilibrium number of criminals by assuming incorrect expectations as to the risk of getting caught.

${ }^{22}$ Since our model is not cast in continuous time, equation (A1) should actually be formulated as a difference equation. However, if the absolute value of $\beta$ is very small the result of the stability analysis will be the same. Even if the discrete model produces over-shootings, there will be convergence.
} 
Here, $\dot{\hat{\delta}}$ is the time derivative of $\hat{\delta}$ and $\beta$ is a negative constant reflecting the sensitivity of $\hat{\delta}$ to a discrepancy between $V_{w}$ and $V_{c}$. Equation (A1) thus stipulates that if the utility of the worker at $\hat{\delta}$ is higher than that of the criminal, the number of criminals should decrease. For stability, a fall in the number of criminals should then, via the consequent changes of $\mu$ and $p$ (equations (4), (5) and (11) are satisfied all the time) close the gap between $V_{w}$ and $V_{c}$.

Just as for the case of the stationary equilibrium system, we have solved the system numerically. The procedure was to change the value of $\hat{\delta}$ somewhat in comparison to the stationary equilibrium value. The probabilities $\mu$ and $p$ were then adjusted to satisfy equations (4), (5) and (11). We then focused on the question whether this leads to a positive or a negative gap between $V_{w}$ and $V_{c}$. It turns out that the low-crime equilibria in Figure 1 are stable, but not the medium-crime equilibria. Inside the convex set depicted in Figure 1, $\hat{\delta}$ will fall. But outside the convex set, crime increases, as is shown by the arrows in the figure. Consequently there are two stable equilibria. One is the situation where everybody is a crook and no honest work is done, i. e., $\hat{\delta}=1$. The other stable equilibrium is the low-crime equilibrium. In between there is an unstable medium-crime equilibrium.

\section{Equilibrium dynamics}

The above discussion started from a fairly self-evident disequilibrium experiment. What happens if the level of criminality is too low or too high in comparison to the stationary equilibrium? Alternatively we could ask what would happen if some parameter changes, for example the tax rate, but the new stationary equilibrium is approached under equilibrium. The trajectory to the new stationary equilibrium is affected by history since cohorts of internees inhabit prisons and these cohorts are a consequence of previous criminal policy. Even if everybody (as in intertemporal equilibrium) has correct expectations as to the present and future values of $\hat{\delta}, \mu$ and $p$ we would not expect an immediate convergence to the new stationary equilibrium.

However, we have chosen not to anlyze the rational-expectations trajectory from an original to a new stationary equilibrium. Here individuals need to have correct 
information about the endogenous variables $\hat{\delta}, \mu$ and $p$ for the present period as well as for future periods (intertemporal equilibrium). Instead we assume that the individual has correct information about $\hat{\delta}, \mu$ and $p$ for the present period, but not necessarily for future periods. We consequently analyze a string of temporary equilibria. But temporary equilibrium is not defined unless we specify a function generating expectations for the endogenous variables in future periods. There are many possibilities, but a rather obvious candidate would be that individuals (wrongly) think that the present period constitutes a stationary equilibrium.

We can thus in principle start from the stationary equilibrium system (3), (4), (5) and (11), but we have to make a correction for the fact that the number of internees is not described by the number of criminals caught during a period, times the average term in prison. Instead we have to start from the following equation where the number of internees equals the number of criminals caught during each of the previous $n$ periods:

$$
i_{t}=\sum_{j=1}^{n} p_{t-j} \mu_{t-j}\left(\hat{\delta}_{t-j}-i_{t-j}\right)
$$

Before we can use this equation we have to solve a conceptual problem. Will old internees be subject to the laws ruling before criminal policy was changed or will the terms be determined by the new policy? The most natural solution would be to refer to the principle of no retroactive legislation. Old internees would therefore be treated according to the old laws, at least if the new laws were more severe than the old ones. However, to eliminate the need to analyze parallel law systems during transitions we assume that the new laws determine the conditions even for $n$ and $f$. The remaining policy parameter, $t$, constitutes no problem from a principle point of view.

A number of simulations give the following non-surprising results. First of all, under temporary equilibrium, the historically determined cohorts of prisoners drive dynamics. A small parameter change (for example in $f$ ) will not immediately result in a new stationary equilibrium, but convergence will be rather quick. Secondly, whether a low-crime or a medium-crime equilibrium will be approached after a disturbance wholly depends on expectations and not on the starting point. Starting from a low- 
criminality equilibrium is no guarantee that a new low-criminality equilibrium will be approached. But if expectations are heavily influenced by the values of the endogenous variables of the old stationary equilibrium, the process will converge to a new equilibrium in the neighborhood of the old one. This result also says something about the choice between the three different equilibria. Abstracting from the inertia created by the cohorts of prisoners, we can jump from one stationary equilibrium to another (for example from a stable low-level to an unstable medium-level criminality equilibrium) if the expectations of all agents are instantly changed to conform to the new equilibrium. 


\section{References}

Becker, Gary S., "Crime and Punishment: An Economic Approach", Journal of Political Economy 1968, 76, 169-217.

Block, M. K.and J.M. Heineke, "A Labor Theoretic Analysis of the Criminal Choice”, American Economic Review, 1975, 65, 314-325.

Carr-Hill, R. A. and Nicholas Stern, "An Econometric Model of Supply and Control of Recorded Offences in England and Wales", Journal of Public Economics, 1973, 2, 289-318.

Davis, Michael L., "Time and Punishment: An Intertemporal Model of Crime", Journal of Political Economy, 1988, 96, 383-390.

Dilulio, John J. Jr, "Help Wanted: Economists, Crime and Public Policy”, Journal of Economic Perspectives, 1996, 10, 3-24.

Ehrlich, Isaac, "Participation in Illegal Activities: A Theoretical and Empirical Investigation", Journal of Political Economy, 1973, 81, 521-565.

Ehrlich, Isaac, "On the Usefulness of Controlling Individuals: An Economic Analysis of Rehabilitation, Incapacitation and Deterrence", American Economic Review, 1981, $71,307-322$.

Ehrlich, Isaac, "Crime, Punishment, and the Market for Offenses", Journal of Economic Perspectives, 1996, 10, 43-67.

Fender, John, “A General Equilibrium Model of Crime and Punishment”, Journal of Economic Behavior and Organization, 1999, 39, 437-453.

Freeman, Richard B., "The Economics of Crime", in O. Aschenfelter and D. Card (eds.), Handbook of Labor Economics, Vol. III, Amsterdam and New York: NorthHolland Elsevier 1999. 
Friedman, David, "Why Not Hang Them All: The Virtues of Inefficient Punishment", Journal of Political Economy, 1999, 107, S259-S269.

Furlong, William J., “A General Equilibrium Model of Crime Commission and Prevention", Journal of Public Economics, 1987, 34, 87-103.

Langan, Patrick A. and David P. Farrington, Crime and Justice in the United States and in England and Wales, 1981-96, U.S. Department of Justice 1998.

Murphy, Kelvin M., Andrei Shleifer and Robert W. Vishny, "Why is Rent-Seeking So Costly to Growth?", American Economic Review, 1993, 83, No 2, 409-414.

Phillips, Llad, and Harold Votey, "Crime control in California", Journal of Legal Studies, 1975, 4, 327-349.

Polinsky, A. Mitchell, and Steven Shavell, "The Economic Theory of Public Enforcement of Law", Journal of Economic Literature, 2000, 38, 45-76.

Sah, Raaj K., "Social Osmosis and Patterns of Crime", Journal of Political Economy, 1991, 99, 1272-1295.

Shavell, Steven, "A Model of Optimal Incapacitation", American Economic Review, $1987,77,107-110$.

Statistics Sweden: Statistisk Årsbok för Sverige 2001 (Statistical Yearbook of Sweden), SCB, Stockholm 2001. 


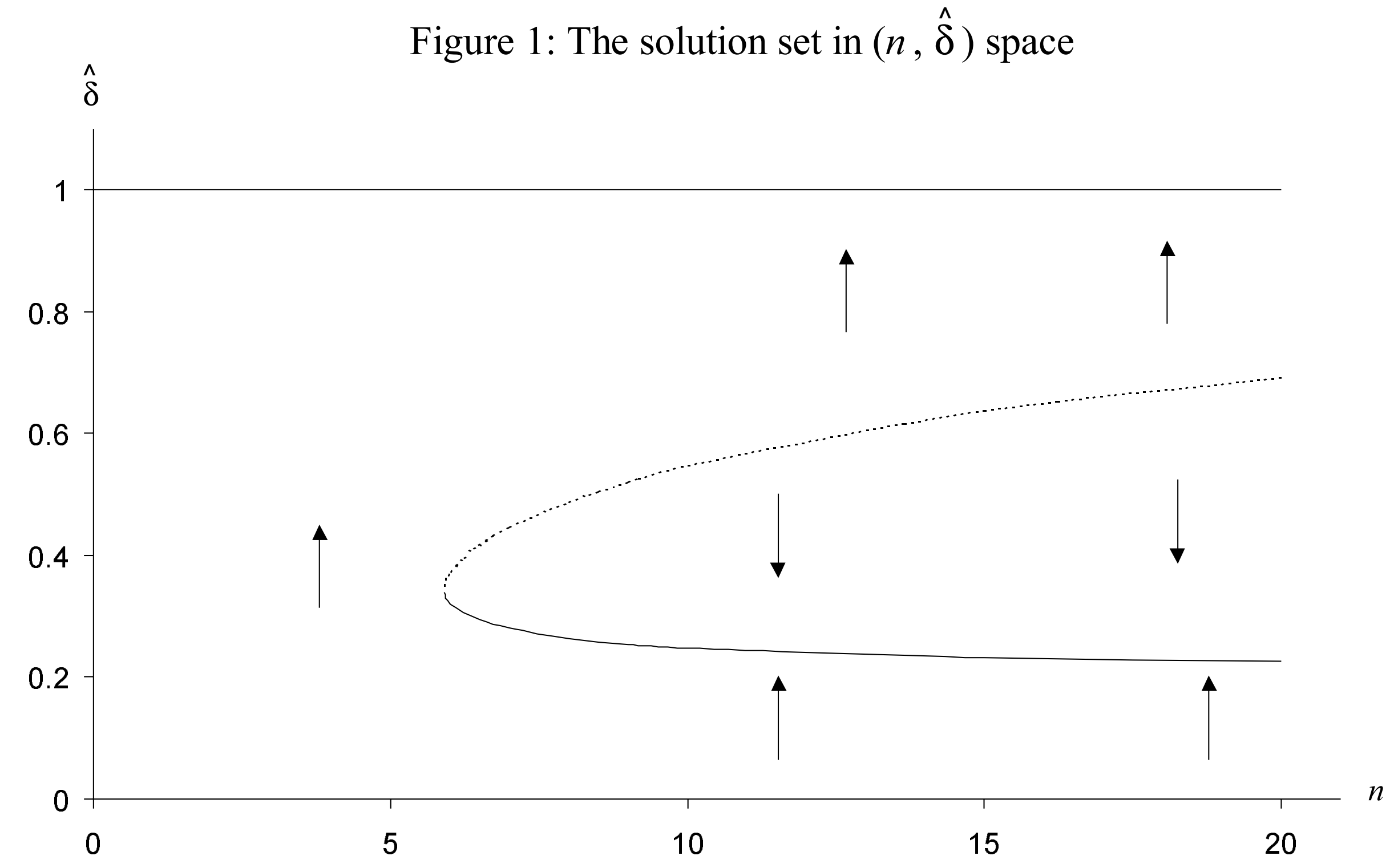


Figure 2: $\hat{\delta}$ and $c r$ isoquants in $(n, f)$ space

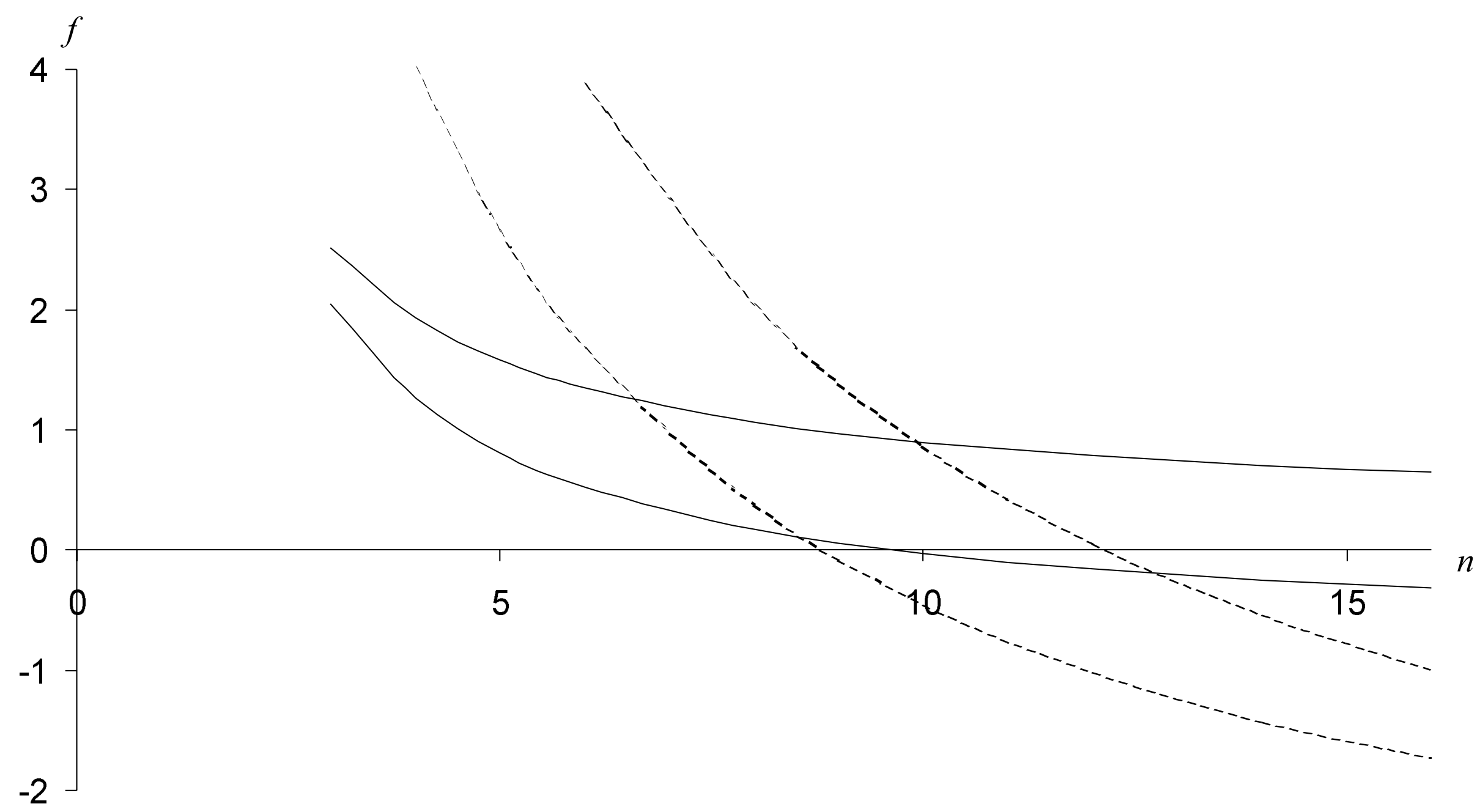

